\title{
Ophthalmic Drug Discovery and Development
}

\author{
Hovhannes J. Gukasyan ' (D) - Shumet Hailu ' Thomas K. Karami '
}

Received: 7 March 2019 / Accepted: 7 March 2019 / Published online: 18 March 2019

(C) Springer Science+Business Media, LLC, part of Springer Nature 2019

Ophthalmic Drug Discovery and Development (ODDD) involves scientific and technological efforts by academic and industrial institutions for developing novel ocular therapeutics to fulfill the unmet needs in treatments of eye diseases. Ophthalmic therapeutics along with vision care are vital aspects of health care worldwide due to their impact on the quality of life for patients with ocular disorders. The objective of this Pharmaceutical Research topical collection is to provide an overview of the existing knowledge in ODDD and present innovative progresses made within Ophthalmic Research and Development (R\&D).

With approval of the first ocular gene therapy product in the United States, Luxturna ${ }^{\mathrm{TM}}$ (voretigene neparvovec-rzyl; Spark Therapeutics, Inc.) in Dec. 2017, a new era of ophthalmic research has started for finding fundamental cures for inherited retinal diseases (IRD). The novel ocular gene therapy product, Luxturna, delivers copy of the RPE65 gene to retinal cells, essentially to cure retinal dystrophy. This innovative approach has created optimism for scientists within the field of ophthalmology in considering gene therapy as mainstream treatment for IRD. A significant theme in this special issue examines IRD, wherein Ong et al. and Rodrigues et al. present comprehensive reviews on development of AAVbased (adeno-associated vector) gene therapies to provide an overview on design and development of ocular products for treatment of IRD using this unique therapeutic modality. The

Hovhannes J. Gukasyan

hovhannes.gukasyan@allergan.com

Shumet Hailu

shumet.hailu@allergan.com

Thomas K. Karami

thomas.karami@allergan.com

Allergan plc., Pharmaceutical Development, Irvine, California, USA article by Rodrigues et al. outlines a broad overview of retinal diseases, vector selection and characterization, AAV production, formulation development strategies, and regulatory aspects of gene therapy. Furthermore, Benjamin Yerxa, CEO of Foundation Fighting Blindness, presents a perspective on the progress made within drug discovery and development for treatment of IRDs, highlighting a substantial shift from reformulating compendial systemic drugs for ocular use to novel discovery approaches in conjunction with revolutionized molecular biology, genetic sequencing and gene therapy, where the supportive role of nonprofit foundations in advancement of new therapies into approved ophthalmic products showcases collaborative and innovative funding models.

Translational preclinical pharmacologic models are important for understanding of eye disease etiology, pathology and evolutions to enable development of new ophthalmic drugs. Shah $e t a l$. in their article provide an extensive review on preclinical animal models utilized in the discovery and development of new therapies for ocular diseases such as dry eye syndrome, glaucoma, presbyopia and retinal disorders e.g. age-related macular degeneration (AMD) and diabetic retinopathy. Use of prognostic or predictive biomarkers in nonclinical and translational research is essential to understand fundamentals in ocular diseases such as diagnosis, disease modulation and monitoring of response to ophthalmic drugs. Biomarkers are measurable indicators of healthy or pathological states for drug evaluation within clinical settings. Tamhane et al. in an inclusive review article on biomarkers in ocular matrices have discussed the advantage of biomarker applications in ophthalmic drug discovery and development, where their utility in commonly sampled ocular matrices (tears, conjunctiva, aqueous humor and vitreous) is broadly discussed. Considering translational medical viewpoint, a perspective article by Rodrigues et al. highlights challenges with viability of using small animal models, as reported in literature, for evaluation of topical delivery of small or large molecule therapeutics to the back of the eye, which may not present 
a direct proxy to larger species or humans. The authors highlight importance of persisting issues, i.e. low bioavailability upon topical dosing, and critically discuss future directions in the ophthalmic drug delivery, with an emphasis on deep understanding of translational science.

Preclinical safety assessment of ophthalmic excipients used for intravitreal injections (IVT) is essential for development of intravitreal products, a topic on which Aguirre et al. report a systematic safety assessment. This research paper considers numerous key characteristics in liquid formulation vehicles following IVT administration in rabbits. Aguirre et al. identify several vehicles that were welltolerated and feasible candidates for novel product development in future intravitreal investigations.

Preservatives have an important role in stability and safety of ophthalmic liquid formulations. Historically used preservatives, benzalkonium chloride (BAC) and polyquaternium-1 (PQ1), are known to have tolerability issues in patients. Von Deylen et al. report their thorough research on synthesizing new generation(s) of polycationic preservatives (pyridine- and piperidine based structures) with high antimicrobial activities against bacteria and fungi and a better tolerability profile. This is a significant advancement for sterility of ophthalmic products from a microbiology point of view, as there have not been many novel chemical preservative compositions introduced in the global ophthalmic market within the last decade. The authors tested their synthetized polycationic preservatives for tolerability and activity, with results showing high antimicrobial effectiveness against relevant microorganisms, noninferiority to BAC and PQ1, with significantly better tolerability profiles.

Understanding similarities and differences between animal models and patients (non-clinical vs. clinical), and subject demographics is essential in Ophthalmic R\&D, as there are multiple physiologically important parameters for targeted ocular drug delivery that need to be judiciously considered. Shen et al. comprehensively present targeted ocular drug delivery with respect to pharmacokinetic $(\mathrm{PK}) /$ pharmacodynamic (PD) considerations in an exclusive article that provides an overview on fundamental approaches used for development of novel ophthalmic medications, i.e. ophthalmic formulation platforms (solutions, suspensions, emulsions, ointments), evaluation of drugs' permeability or enhancement thereof, screening of physicochemical properties, along with design of sustained release intraocular drug delivery systems (e.g. intraocular implants). Furthermore, physicochemical characterization and evaluation of drug release profiles of ophthalmic formulations is equally important. Bao and Burgess elegantly discuss perspectives on physical and chemical characterization of ophthalmic ointments, and the unique combination of features that these special class of semisolid drug delivery systems offer. In vitro release testing methods from semisolid ophthalmic drug products is critically discussed from a sound fundamental pharmaceutics and a pragmatic point of view. From drug delivery perspective, biodegradable polymers and biopolymers have a central function for sustained release ocular delivery. Tran et al. in an expert review discuss an emerging role for silk to be considered for ophthalmic drug delivery. Silk is an outstanding biopolymer being used for load-bearing applications, including surgical threads and meshes, which are clinically approved for use in human. The review emphasizes emerging trends for use of silk as a biopolymer in ocular therapeutics.

Primary open angle glaucoma (POAG) remains as the leading cause for irreversible blindness. Treatment of glaucoma has been one of the enduring challenges within ODDD. Traditional formulae for glaucoma therapeutics as eye drops have shown compliance issues within patient demographics, which is the most common cause for the "treatment failure". Adams et al. provide an excellent expert review on glaucoma and the next generation therapeutics for treatment of the disease. The authors highlight that the recent approval of two new intraocular pressure (IOP)-lowering drugs; (Vyzulta ${ }^{\mathrm{TM}}$, $0.024 \%$ latanoprostene bunod) and Rhopressa ${ }^{\mathrm{TM}}(0.02 \%$ netarsudil) with distinct mechanisms of action, are the first in over 20 years that have been introduced to the market. While these new IOP reducing medications are still administered as topical eye drops, to increase patient compliance and achieve a higher therapeutic benefit, development of sustained release delivery technologies for treatment of POAG is critically reviewed. Several innovative intraocular, sustained release implants designed to lower the IOP in a durable fashion are emphasized. Furthermore, a new approach explored by ophthalmic drug discovery and development teams as alternative for glaucoma therapy with focus on neuroprotection is discussed, which includes IOP agnostic preservation of the retinal ganglion cells and optic nerve.

A few advanced topics in regulatory science and strategy as they relate to ODDD are assessed within the thematic collection by Rowe-Rendleman and Harigaya et al. Patient reported outcomes (PROs), a significant and imperative novel concept advocated by regulatory agencies and government run research institutions, is skillfully introduced by RoweRendleman in an expert review which explains PRO as it applies to ODDD. New guidances delineate traditional regulatory commitments and methodology from implementing structured assessment of risk vs. benefit in clinical trials, to additionally develop reliable and validated tools that measure patients' experience. Bioequivalence in topical ophthalmic formulations can be evaluated by PK drug exposure in aqueous humor $(\mathrm{AH})$. Harigaya et al. present an overview of in vivo human AH PK studies submitted for regulatory consideration that utilize common ophthalmic corticosteroid suspensions. Their bioequivalence study methods with PK endpoints suggest that differences in ethnicity and age of the studied 
population play important roles in ocular exposure profiles of corticosteroids. Therefore, to identify true effects from formulation differences in designing bioequivalence studies, the subject-specific covariate influences need to be eliminated. Lastly, impact of ophthalmic formulation composition on ocular tissues exposure and distribution is presented by Mandal et al. in a thorough review article. An exceptional comparecontrast in the aforementioned article, from a relative ophthalmic bioavailability perspective, provides insight on formulation development, preclinical and clinical pharmacokinetic performance of a nano-micellar topical ophthalmic solution of cyclosporine.

The reviews, research articles and perspectives presented in this ophthalmic theme issue are great examples of new, inno- vative studies, ideas, and essential knowledge in the field of ODDD collected from contributions by expert academic and industrial peers within Ophthalmic R\&D. We hope this theme issue will provide a good fundamental overview of status quo and will significantly impact expansion of ongoing research and development within ODDD. This collection is a testament to academic and industrial researchers who are eagerly searching for safe and effective treatments for challenging ophthalmic diseases, moreover we hope it can serve as a gateway for innovation and resources to newcomers in the field of ophthalmic drug discovery and development.

Publisher's Note Springer Nature remains neutral with regard to jurisdictional claims in published maps and institutional affliations. 